\title{
The tumour microenvironment of the upper and lower gastrointestinal tract differentially influences dendritic cell maturation
}

\author{
Maria E. Morrissey¹, Róisín Byrne', Celina Nulty', Niamh H. McCabe, Niamh Lynam-Lennon', Clare T. Butler², \\ Susan Kennedy ${ }^{1}$, Dermot OToole ${ }^{3}$, John Larkin ${ }^{4}$, Paul McCormick ${ }^{4}$, Brian Mehigan ${ }^{4}$, Mary-Clare Cathcart ${ }^{1}$, \\ Joanne Lysaghtt, John V. Reynolds ${ }^{1,5}$, Elizabeth J. Ryan ${ }^{6,7}$, Margaret R. Dunne $^{1}$ and Jacintha O'Sullivan ${ }^{1 *}$ (D)
}

\begin{abstract}
Background: Only 10-30\% of oesophageal and rectal adenocarcinoma patients treated with neoadjuvant chemoradiotherapy have a complete pathological response. Inflammatory and angiogenic mediators in the tumour microenvironment (TME) may enable evasion of anti-tumour immune responses.

Methods: The TME influence on infiltrating dendritic cells (DCs) was modelled by treating immature monocyte-derived DCs with Tumour Conditioned Media (TCM) from distinct gastrointestinal sites, prior to LPS-induced maturation.

Results: Cell line conditioned media from gastrointestinal cell lines inhibited LPS-induced DC markers and TNF-a secretion. TCM generated from human tumour biopsies from oesophageal, rectal and colonic adenocarcinoma induced different effects on LPS-induced DC markers - CD54, CD80, HLA-DR, CD86 and CD83 were enhanced by oesophageal cancer; CD80, CD86 and CD83 were enhanced by rectal cancer, whereas CD54, HLA-DR, CD86, CD83 and PD-L1 were inhibited by colonic cancer. Notably, TCM from all Gl cancer types inhibited TNF-a secretion. Additionally, TCM from irradiated biopsies inhibited DC markers. Profiling the TCM showed that IL-2 levels positively correlated with maturation marker CD54, while Ang-2 and bFGF levels negatively correlated with CD54.
\end{abstract}

Conclusion: This study identifies that there are differences in DC maturational capacity induced by the TME of distinct gastrointestinal cancers. This could potentially have implications for anti-tumour immunity and response to radiotherapy.

Keywords: Gastrointestinal cancer, Dendritic cell inhibition, Tumour conditioned media, Tumour microenvironment, Radiotherapy, TNF-a

\section{Background}

The 5-year overall survival rates across gastrointestinal (GI) cancer types vary, with oesophageal adenocarcinoma (OAC) low at $18-19 \%$, whereas colonic and rectal adenocarcinoma rates stand at $58-59 \%$ according to the

\footnotetext{
* Correspondence: osullij4@tcd.ie

${ }^{1}$ Department of Surgery, Trinity Translational Medicine Institute, Trinity College Dublin, St James's Hospital, Dublin 8, Ireland

Full list of author information is available at the end of the article
}

National Cancer Registry Ireland [1]. OAC rates have increased by almost half in recent years in Western countries, mirroring the increase in obesity [2]. Colorectal cancer (CRC) is the third most common cancer worldwide with rectal cancer accounting for approximately 35\% of CRC cases. Typically, epidemiological and scientific studies group colon and rectal cancer together, despite their different standard treatment regimens [3, 4]. Standard treatment for oesophageal and rectal adenocarcinoma

C C The Author(s). 2020 Open Access This article is licensed under a Creative Commons Attribution 4.0 International License, which permits use, sharing, adaptation, distribution and reproduction in any medium or format, as long as you give appropriate credit to the original author(s) and the source, provide a link to the Creative Commons licence, and indicate if changes were made. The images or other third party material in this article are included in the article's Creative Commons licence, unless indicated otherwise in a credit line to the material. If material is not included in the article's Creative Commons licence and your intended use is not permitted by statutory regulation or exceeds the permitted use, you will need to obtain permission directly from the copyright holder. To view a copy of this licence, visit http://creativecommons.org/licenses/by/4.0/. The Creative Commons Public Domain Dedication waiver (http://creativecommons.org/publicdomain/zero/1.0/) applies to the data made available in this article, unless otherwise stated in a credit line to the data. 
involves neoadjuvant chemoradiotherapy (CRT) to shrink the tumour prior to surgical resection, whereas for colonic adenocarcinoma the standard treatment involves surgical resection followed by adjuvant targeted therapies [5-7]. Response to CRT is highly variable with just $10-30 \%$ of patients achieving a complete pathological response, which is linked with higher 5-year survival rates, for both oesophageal and rectal cancer [8-13]. Tumours have different levels of radiosensitivity and a number of cellular processes and immune mechanisms have been implicated in radioresponse phenotypes [14-16]. Understanding the key components of the immune system which are modulated by the tumour microenvironment (TME) may offer insights into ways to improve the clinical outcome for patients with GI cancers by identifying either prognostic biomarkers or novel therapeutic strategies.

Dendritic cells (DCs) are professional antigenpresentation cells responsible for activation of $\mathrm{T}$ cells and thus orchestration of the adaptive immune response [17]. Immature DCs recognise and capture antigens and are characterised by low expression of maturation and costimulatory markers such as CD83, CD54, CD80, CD86; HLA-DR for antigen presentation and other immunoinhibitory markers such as PD-L1 $[18,19]$. DC maturation, a crucial factor for efficient $\mathrm{T}$ cell activation, is triggered in response to various inflammatory mediators and TLRdependent activation, such as bacterial LPS via TLR4, leading to the increased expression of several cell surface markers by DCs, migration to lymph nodes and presentation of antigens via MHC class I and II molecules to activate $\mathrm{CD}_{4}^{+}$and $\mathrm{CD}^{+}{ }^{+} \mathrm{T}$ cells [20]. Factors such as IL-10 and VEGF in the TME influence DC function and these can inhibit IL-12p70 and TNF- $\alpha$ production from DCs $[21,22]$. DCs which secrete high levels of IL-12p70 induce anti-tumour immunity, as they have increased capacity to enhance natural killer cell activity, skew $\mathrm{T}$ cell responses to $\mathrm{T}$ helper (Th)-1 type and prime tumour antigen specific $\mathrm{T}$ cells $[23,24]$. Decreased IL-12p70 expression is associated with suppressed endocytic activity and antigenpresentation machinery, and also decreased motility of anti-tumour immune cells to the tumour site [25]. TNF- $\alpha$ released by immunostimulatory DCs can also act to enhance $\mathrm{T}$ cell stimulatory capacity, while increasing IL-12 production from DCs and decreasing production of the immunosuppressive cytokine IL-10 [26, 27].

Known risk factors for the development of GI cancers include inflammatory disorders, specifically Barrett's oesophagus for OAC and inflammatory bowel disease for CRC [28-30]. Not only is inflammation a hallmark of cancer, it plays a pivotal role in modulating radiation responsiveness of tumours [31]. Radiation can elicit the systemic release of the TLR ligands, damage-associated molecular patterns (DAMPs), after oesophageal irradiation or locally after targeted tumour irradiation, such as treatment for CRC [32-37]. TLR-dependent activation of DCs after irradiation supports the use of low dose hypofractionated radiotherapy as an adjuvant to immunotherapy to enhance its effect, however either very low or high levels may be immunosuppressive [32]. Direct immunomodulatory effects of irradiation on immune cells have been reported, such as altered IL-12 production from DCs $[33,34]$. It is important to understand the immunosuppressive nature of the TME for tumourinfiltrating DCs, which may limit the success of different treatments, e.g. immunotherapies, including DC vaccines [38].

We have previously described the immunosuppressive effect of the colonic TME which inhibits LPS-induced DC maturation [18, 20, 39-41]. Using a similar experimental outline as we described previously, in this study we investigated the effects on DC maturational capacity across different human GI cancers, using conditioned media from cell lines (in vitro conditioned media) and treatment-naïve tumour biopsies (ex vivo TCM) (Supplementary Fig. $1 \mathrm{~A}$ ). While in vitro conditioned media represents the secretome from cancer epithelial cells, ex vivo conditioned media is a more complex model containing the soluble contributions from many different cells within the tumour microenvironment $[18,20,39-$ 41]. Due to our interest in understanding the tumour microenvironment in both upper and lower GI tract cancers; oesophageal, rectal and colonic adenocarcinoma were investigated. This study describes for the first time that there were unexpected differences induced by the TCM on maturation of monocyte-derived DCs. Here, oesophageal cancer induced the highest level of DC maturation markers, rectal cancer induced moderate levels of DC maturation markers and colonic cancer significantly inhibited DC maturation markers. Interestingly for all GI cancer types examined here, in vitro and ex vivo TCM significantly inhibited TNF- $\alpha$ secretion from DCs. In addition, we modelled radiotherapy of oesophageal and rectal biopsies and found that TCM from 2Gy-irradiated tumours inhibited LPS-induced DC markers. Differential levels of specific inflammatory and angiogenic mediators were detectable in ex vivo TCM of GI cancers that correlated with DC maturation.

\section{Methods}

\section{Cell culture and irradiation}

Human oesophageal adenocarcinoma cell lines - isogenic OE33 parental (OE33 P) and radioresistant (OE33 R) lines [42], and commercially available colorectal adenocarcinoma - SW480 and SW620 lines, were maintained in RPMI-1640 medium (Invitrogen) supplemented with $10 \% \mathrm{v} / \mathrm{v}$ FBS and $1 \% \mathrm{v} / \mathrm{v}$ penicillin/streptomycin in a humidified atmosphere with $5 \% \mathrm{CO}_{2}$ at $37^{\circ} \mathrm{C} .3 \times 10^{5}$ cells in $2 \mathrm{ml}$ media were seeded in 6-well plates, allowed 
adhere overnight and were 0Gy- (mock) or 2Gyirradiated at $70 \%$ confluence using an X-Ray generator (RS 225 system, using X-rays from a Tungsten target at a rate of $3.25 \mathrm{~Gy} / \mathrm{min}$ ) (Gulmay Medical, UK). Following 24-h culture, the supernatant was harvested and frozen as in vitro conditioned media from three independent biological replicates.

\section{Human ex vivo tumour explant culture and irradiation}

All tissue was obtained with prior written informed consent from each patient, and ethical approval was granted by the Adelaide and Meath Hospital (AMNCH), Tallaght, Dublin Research Ethics Committee. All biopsy samples used were treatment-naïve (Supplementary Table 1). Patient-matched tumour biopsies taken from patients with oesophageal or rectal adenocarcinoma were either 0Gy- (mock) or 2Gy-irradiated using an XRay generator (RS 225 system, using X-rays from a Tungsten target at a rate of $3.25 \mathrm{~Gy} / \mathrm{min}$ ) (Gulmay Medical, UK). For oesophageal adenocarcinoma, fresh tissue was cultured for $24 \mathrm{~h}$ in complete M199 (cM199) M199 media (Invitrogen) media supplemented with 10\% $\mathrm{v} / \mathrm{v}$ FBS, $1 \% \mathrm{v} / \mathrm{v}$ penicillin/streptomycin and $1 \mu \mathrm{g} / \mathrm{ml}$ insulin (Sigma-Aldrich UK). For rectal adenocarcinoma samples, fresh tissue was washed and cultured for $48 \mathrm{~h}$ in complete RPMI (cRPMI) - RPMI-1640 (Invitrogen) media supplemented with 10\% FBS, 1\% penicillin/ streptomycin, $1 \%$ fungizone and $50 \mu \mathrm{g} / \mathrm{ml}$ gentamicin. For colonic adenocarcinoma, the tissue was cultured for $72 \mathrm{~h}$ in cRPMI - RPMI-1640 media (Invitrogen) supplemented with $10 \%$ FBS, $1 \%$ penicillin/streptomycin and $1 \%$ fungizone. Culturing was performed in a humidified atmosphere with $5 \% \mathrm{CO}_{2}$ at $37^{\circ} \mathrm{C}$. Ex vivo TCM was harvested and frozen at $-80^{\circ} \mathrm{C}$.

\section{DC isolation and culture}

Human monocyte-derived immature DCs were generated from peripheral blood mononuclear cells (PBMCs) obtained from buffy coat preparations (National Blood Centre, St. James's Hospital, Dublin) by density gradient centrifugation (Lymphoprep) as we previously described (Supplementary Fig. 1 A) [18, 43]. Briefly, monocytes were isolated by positive selection using anti-CD14 magnetic microbeads as described by the manufacturer (Miltenyi Biotec) and seeded at a density of $1 \times 10^{6}$ cells $/ \mathrm{mL}$ in 6 well plates in $3 \mathrm{~mL}$ of RPMI-1640 medium containing 10\% defined HyClone FBS (Thermo Scientific), 1\% penicillin/streptomycin, $1 \%$ fungizone, human granulocyte macrophage colony-stimulating factor $(50 \mathrm{ng} / \mathrm{mL}$; Immunotools), and human IL-4 (70 ng/mL; Immunotools) in a humidified atmosphere with $5 \% \mathrm{CO}_{2}$ at $37^{\circ} \mathrm{C}$. Cells were fed at day 3 by replacing half the medium made up with

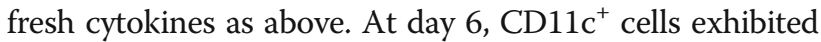

an immature DC phenotype capable of upregulating cell surface markers following LPS activation.

\section{Stimulation of monocyte-derived DCs}

Freshly generated DCs were plated in 96-well plates at $2 \times$ $10^{5}$ cells in $200 \mu \mathrm{L}$ RPMI-1640 media supplemented with 10\% defined Hyclone FBS (Thermo Fisher Scientific) and stimulated with a 1:2 dilution of conditioned media, or matched background media controls, for 4-5 h before exposure to $10 \mu \mathrm{g} \mathrm{ml}-1$ of ultrapure TLR4 agonist Escherichia coli lipopolysaccharide (LPS-EB; Invivogen) overnight. Supernatants were harvested and frozen for ELISA analysis, and cells were assessed for expression of surface markers by flow cytometry as we described previously (Supplementary Fig. 1 A) [18, 20, 39-41].

\section{Flow cytometry}

DCs were stained with the following antibody panel: phycoerythrin (PE)- anti-CD80 (2D10), PerCP-Cy5.5- anti-CD86 (IT2.2), Pe-Cy7- anti-CD83 (HB15), Brilliant Violet 421anti-PD-L1 (29E.2A3), Brilliant Violet 510- anti-CD11c (3.9), allophycocyanin (APC)- anti-CD54 (HA58), and APC-Cy7- anti-HLA-DR (L243) (Biolegend). DC preparations were acquired on DAKO CyAn ADP flow cytometer (Beckman Coulter) with compensation performed with positive and negative antibody capture beads (BD Biosciences). Gating on and analysis of CD11c + cells was performed using FlowJo software (Tree Star Inc.) to determine Mean Fluorescence Intensity (MFI). The gating strategy and Fluorescence Minus One (FMO) staining controls are shown (Supplementary Fig. 1 B-C) and representative histograms are graphed (Supplementary Fig. 2).

\section{Elisa}

Levels of IL-12p70 and TNF- $\alpha$ in DC supernatant were quantified by DuoSet sandwich Enzyme-Linked Immunosorbent Assay (ELISA) kits according to the manufacturer's protocol (R\&D Systems). Multiplex ELISA was performed to simultaneously assess levels of ten inflammatory markers (IL-2, MMP2, MMP9, CCL2, IL-6, CCL20, TNF- $\alpha$, IL-1 $\beta$ and IL-10) or seven angiogenic markers (ICAM-1, VCAM-1, bFGF, VEGF, PAI1 and Ang-2) in ex vivo TCM, according to the manufacturer's protocol (Meso Scale Diagnostics) [44].

\section{Data analysis}

Data displayed in graphs is from one healthy PBMC donor for in vitro TCM $(n=3)$. Data displayed in graphs is from one healthy PBMC donor per cancer type for ex vivo TCM with $n=7-14$ tumour samples as indicated in the legends. Statistical analyses were carried out using GraphPad Prism v5 for Windows (GraphPad software). Paired or unpaired t-test, or ANOVA with post hoc Dunnett's t-tests were used to compare groups as 
indicated. A $p$ value of less than 0.05 was considered to be significant in all of the analyses where $* p \leq 0.05$; ** $p \leq 0.01$ and ${ }^{* * * *} p \leq 0.001$ for t-tests.

\section{Results}

Differential DC maturation induced by conditioned media from OAC and CRC cell lines, while both inhibited TNF-a secretion

We investigated if the maturational capacity of DCs could be influenced by conditioned media harvested from cell lines from upper and lower GI tract cancers. We found that in vitro conditioned media from three independent biological replicates of OAC and CRC cell lines modulated LPS-induced DC maturation (Fig. 1). TCM from the OE33 Radioresistant (OE33 R) cells significantly reduced HLA-DR expression following LPStreatment. Whereas the TCM from both CRC lines SW480 and SW620 inhibited multiple DC surface markers relative to LPS in background media (+) (Fig. 1a). In vitro conditioned media from both SW480 and SW620 lines inhibited HLA-DR, CD86 and CD83 and additionally, SW480 inhibited CD80 and SW620 inhibited CD54. The effect of in vitro conditioned media on IL-12p70 and TNF- $\alpha$ production by DCs was also examined relative to levels induced by LPS in background media (+) (Fig. 1b-c). The conditioned media of both $\mathrm{OAC}$ and CRC lines significantly inhibited LPS-induced TNF- $\alpha$ in DC supernatants (Fig. 1b). No significant changes were observed for LPS-induced IL-12p70 in DC supernatants (Fig. 1c).

In summary, the TCM from OAC cell lines inhibited only HLA-DR on DCs and it inhibited levels of soluble TNF- $\alpha$, whereas the TCM from CRC cell lines inhibited five DC surface markers in addition to soluble TNF- $\alpha$.

\section{TCM from human tumour biopsies induced different effects on DC maturation based on cancer type}

We investigated if the maturational capacity of DCs is influenced by three distinct GI tract cancers. We found that ex vivo TCM from treatment-naïve oesophageal, rectal and colonic adenocarcinoma tissues modulated LPSinduced DC maturation (Fig. 2, Supplementary Fig. 2). TCM from oesophageal adenocarcinoma significantly enhanced CD54 ( $p<0.001)$, CD80 ( $p<0.001)$, HLA-DR $(p=0.001), \mathrm{CD} 86(p<0.001)$ and CD83 $(p<0.001)$ compared to LPS-induction in background media alone, cM199 (+) (Fig. 2a). Moreover, the TCM from rectal adenocarcinoma significantly enhanced CD80 ( $p=0.028)$, CD86 $(p=0.016)$ and CD83 $(p=0.002)$ compared to LPSinduction in background media alone, cRPMI (+) (Fig. 2a). Whereas, the TCM from colonic adenocarcinoma significantly inhibited CD54 ( $p=0.011)$, HLA-DR $(p=0.013)$, CD86 $(p=0.021), \operatorname{CD} 83(p=0.018)$ and PD-L1 $(p=0.006)$ compared to LPS-induction in background media alone,
cRPMI (+). Similar findings were observed in the unstimulated setting, without LPS, where with both oesophageal and rectal TCM, all DC markers were enhanced relative to unstimulated DCs, whereas with colonic TCM, DC markers were at a similar level to unstimulated DCs (Supplementary Fig. 3).

The effect of ex vivo TCM on LPS-induced levels of IL-12p70 and TNF- $\alpha$ in DC supernatants were also examined (Fig. 2b-c). The TCM of oesophageal, rectal and colonic adenocarcinoma significantly inhibited levels of TNF- $\alpha$ in DC supernatants $(p=0.004, p=0.013$ and $p=$ 0.026 respectively) (Fig. 2b). Whereas for IL-12p70, while oesophageal TCM had no effect on LPS-induced levels, rectal TCM significantly enhanced IL-12p70 levels ( $p=0.017)$ and colonic TCM significantly inhibited IL12 p70 levels $(p=0.001)$ compared to LPS-induced levels (Fig. 2c).

Despite some inter-individual variability, ex vivo TCM from oesophageal, rectal and colonic adenocarcinoma differentially primed immature DCs to respond to subsequent LPS stimulation. There were no significant correlations with clinicopathological parameters examined (Supplementary Table 1).

\section{TCM of 2Gy-irradiated TME from GI cancers significantly inhibited LPS-induced DC markers}

As the standard-of-care treatment for oesophageal and rectal cancer includes radiotherapy which influences the immune system through unclear mechanisms, we investigated if the maturational capacity of DCs is influenced by irradiated GI tract cancers. We found that conditioned media harvested from irradiated OAC lines further inhibited LPS-induced DC maturational capacity. While only HLA-DR was significantly inhibited by conditioned media from 0Gy-irradiated OE33 R line compared to LPS-induced levels, CD86, CD80 and PD-L1 were also inhibited by conditioned media from 2Gyirradiated OAC lines (Fig. 1, Supplementary Fig. 4). While most DC markers were significantly inhibited by in vitro conditioned media of 0Gy-irradiated CRC lines, no additional effect was induced by the 2Gy-irradiated CRC lines (Fig. 1, Supplementary Fig. 4).

Similarly, we found that ex vivo TCM generated from biopsies of oesophageal or rectal adenocarcinoma, that received 0 Gy- (mock) or 2Gy-irradiation, differentially altered DC maturational capacity (Fig. 3, Supplementary Fig. 5). In the LPS-stimulated setting, TCM from 2Gy irradiated oesophageal adenocarcinoma biopsies inhibited levels of CD54, compared to 0Gy patient-matched biopsies $(p=0.024)$, whereas, the levels of CD80, HLA-DR, CD86 and PD-L1 were not differentially altered (Fig. 3a). TCM from 2 Gy irradiated rectal adenocarcinoma significantly inhibited LPS-induced DC maturation, compared to 0Gy patient-matched biopsies for CD54 ( $p=0.003)$, 


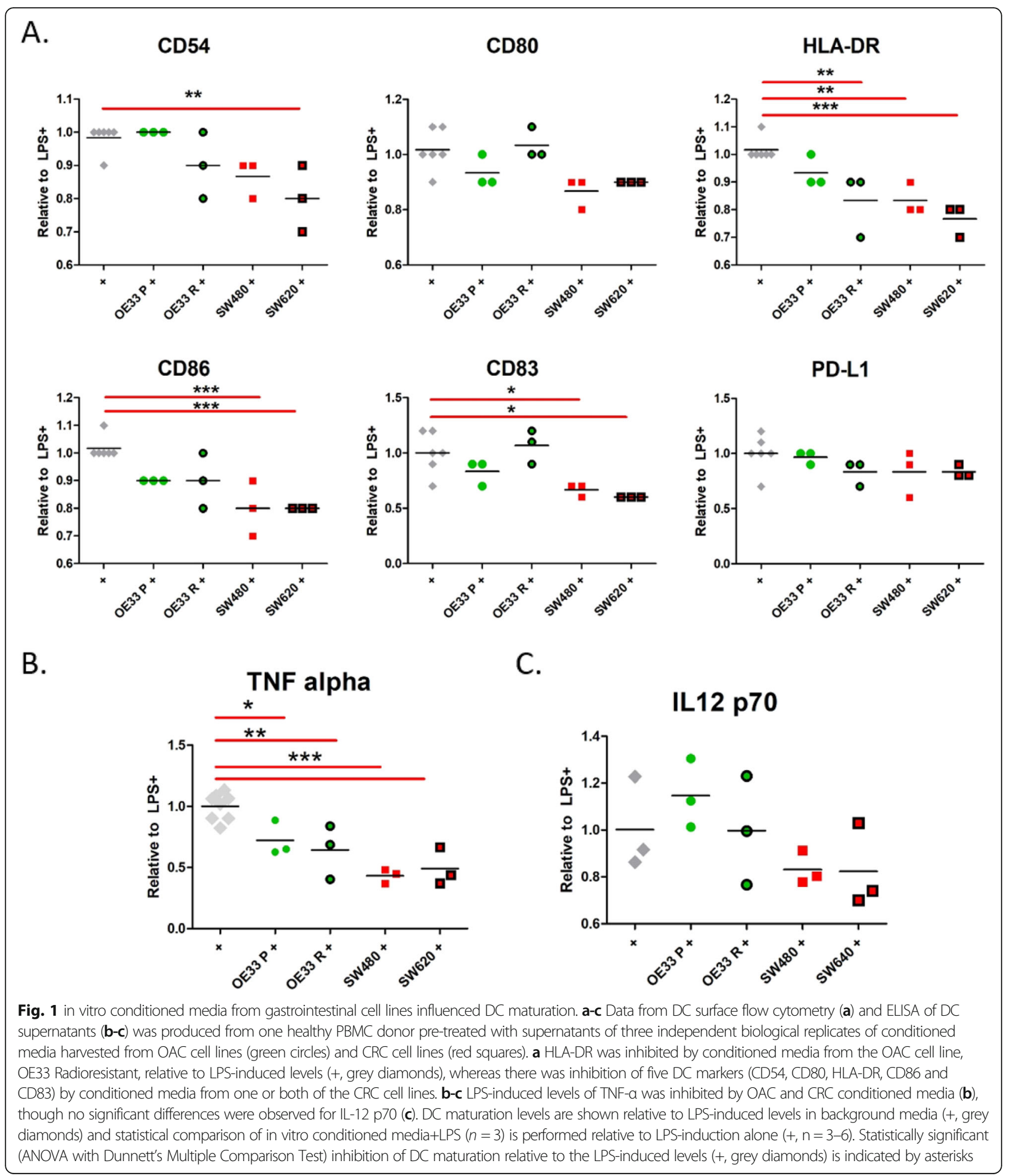

HLA-DR $(p=0.007)$, CD86 $(p=0.050)$ and PD-L1 $(p=0.036)$ (Fig. 3a). There were no differences in LPS-induced levels of TNF- $\alpha$ or IL-12p70 in DC supernatants between 0Gy- and 2Gy-irradiated biopsies for either oesophageal or rectal adenocarcinoma (Fig. $3 b-c)$.
Differential levels of inflammatory and angiogenic mediators in the ex vivo TCM of oesophageal, rectal and colonic adenocarcinoma biopsies correlated with DC maturation marker CD54

As inflammatory and angiogenic mediators influence DCs, we investigated if levels of specific inflammatory 


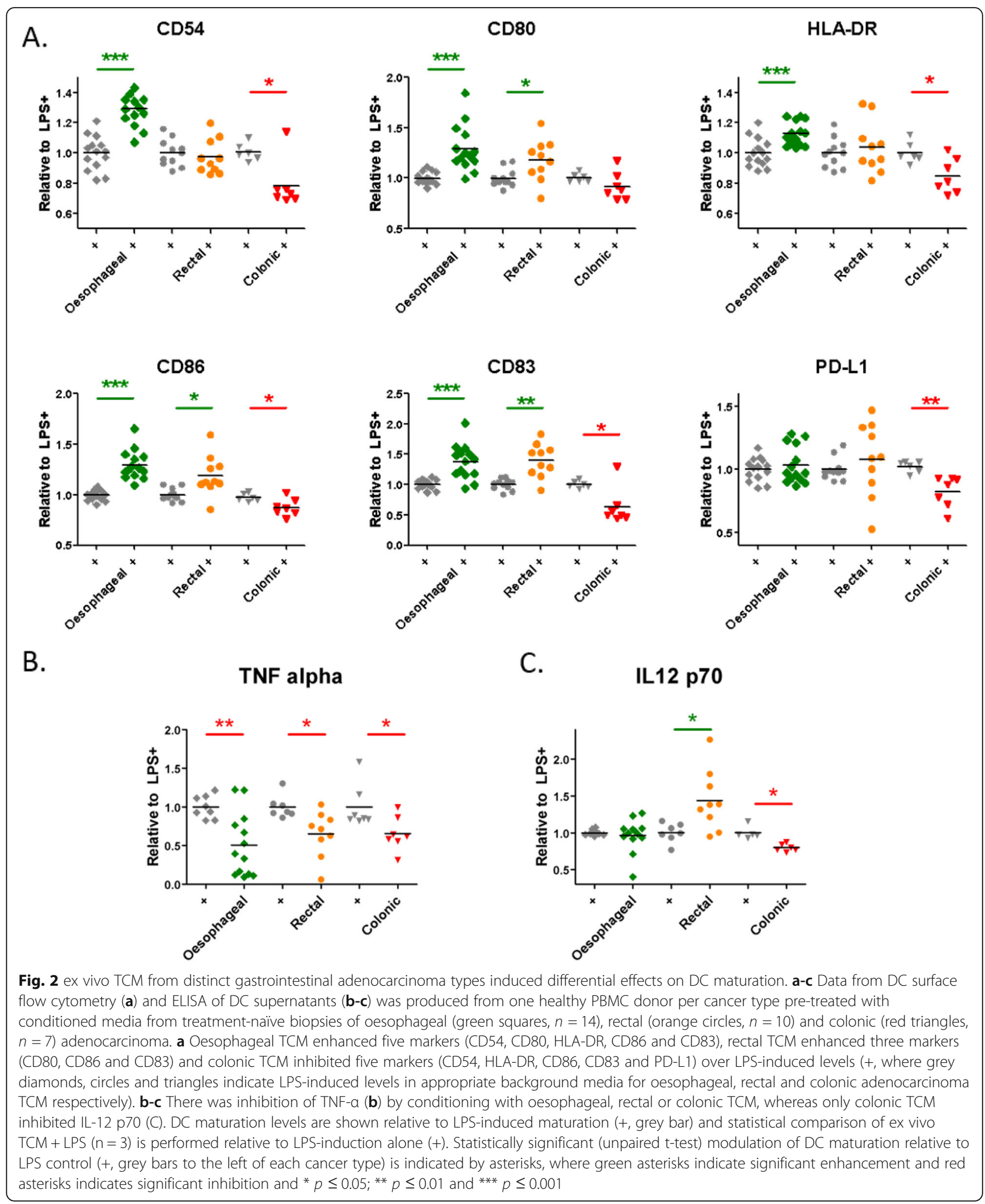




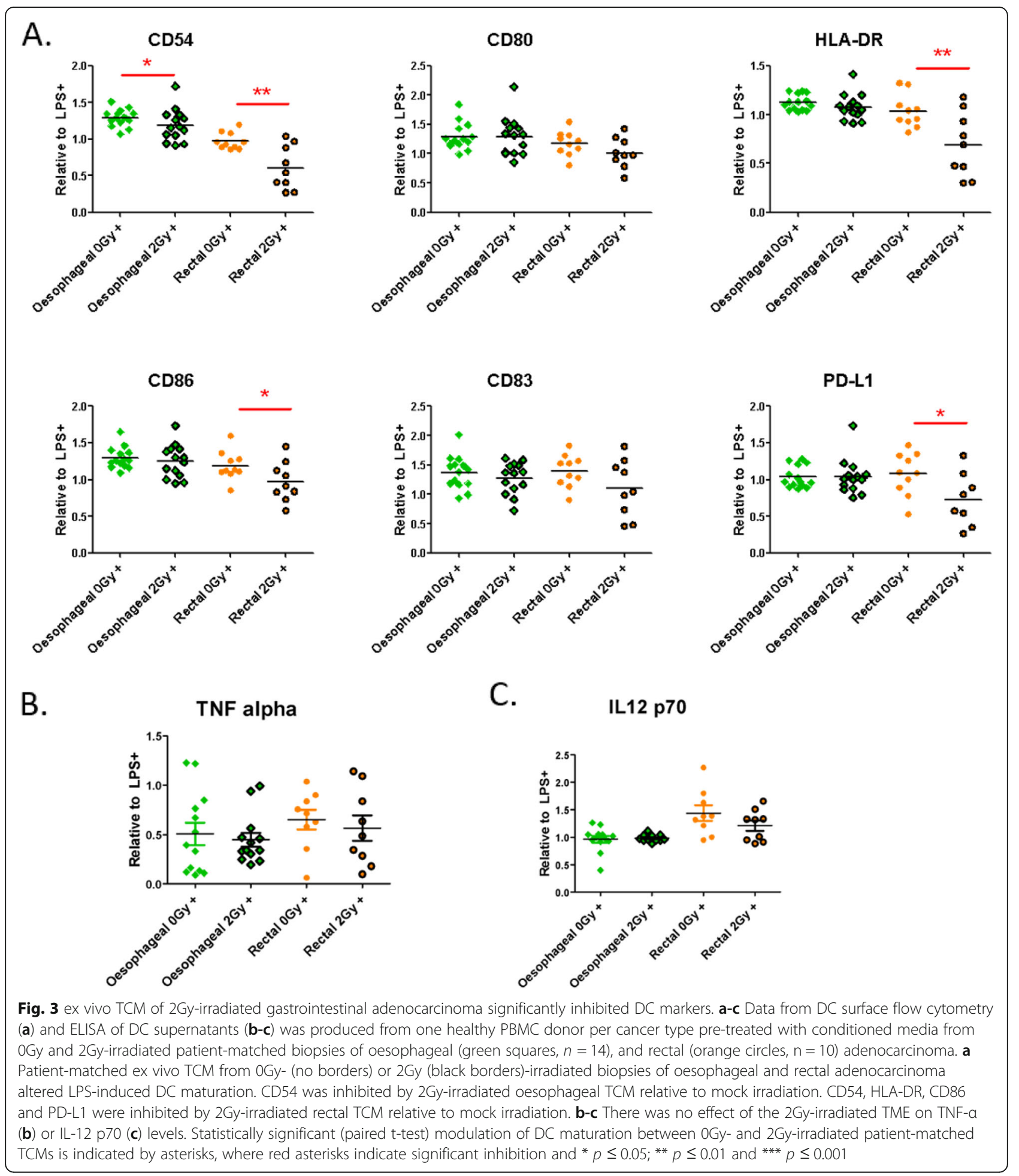

and angiogenic mediators secreted by distinct GI tract cancers correlated with DC inhibition. Ex vivo TCM of oesophageal $(n=14)$, rectal $(n=8)$ and colonic $(n=7)$ human tumour biopsies were screened simultaneously for raw levels of ten inflammatory and seven angiogenic markers. We found that IL-2 was at significantly higher levels in oesophageal than rectal and colonic adenocarcinoma TCM, Ang-2 was at significantly lower levels in oesophageal than colonic TCM and bFGF was at significantly lower levels in oesophageal than rectal and colonic TCM (Fig. 4). Indeed, levels of IL-2, Ang-2 and bFGF across oesophageal, rectal and colonic adenocarcinoma 
correlated with LPS-induced levels of the DC surface marker CD54. Specifically, IL-2 had a Pearson correlation of $\mathrm{r}=0.3(p=0.010)$, Ang- 2 had a Pearson correlation of $\mathrm{r}=-0.3(p=0.010)$ and bFGF had a Pearson correlation of $\mathrm{r}=-0.3(p=0.022)$. The other DC markers - CD80, HLADR, CD86, CD83 and PD-L1, did not show any significant correlations across cancer types (data not shown). Other inflammatory (MMP2, MMP9, CCL2, IL-6, CCL20, TNF- $\alpha$, IL-1 $\beta$ and IL-10) and angiogenic (ICAM-1, VCAM-1, VEGF and PAI1) mediators did not show significant correlations with DC inhibition (data not shown). There were no differences in levels of any of the inflammatory or angiogenic mediators examined between 0Gy- and 2Gyirradiated patient-matched TCMs for either oesophageal or rectal adenocarcinoma biopsies (Fig. 4a-c).

\section{Discussion}

The aim of this study was to examine if DC maturation was influenced by three distinct cancers of the GI tract oesophageal, rectal and colonic adenocarcinoma. As radiotherapy induces unclear effects in terms of immunomodulation, we also investigated the effect of radiotherapy in this setting. The influence of the gastrointestinal TME on infiltrating DCs was modelled by conditioning immature monocyte-derived DCs with TCM, followed by subsequent LPS maturation, to investigate the effect of the TME on the maturational capacity of DCs as previously described [18]. The effect of TCM on LPS-induced levels of DC markers CD54, CD80, HLA-DR, CD86, CD83 and PD-L1, and two secreted cytokines IL-12p70 and TNF- $\alpha$ in DC supernatants, as indicators for DC maturation were examined (Supplementary Fig. $1 \mathrm{~A}$ ). We describe the levels of DC maturational capacity induced by conditioned media from oesophageal and colorectal cell lines. While TCM from OE33 oesophageal cell lines inhibited levels of HLA-DR only, TCM from CRC lines inhibited five DC markers CD54, CD80, HLA-DR, CD86 and CD83. These markers are upregulated on the surface of DCs in order to ensure DCs can stimulate an effective $T$ cell response $[18,19]$. CD83 is the most prominent surface marker for fully matured human DCs and enhances DCs' $T$ cell stimulatory capacity [45]. CD80 and CD86 are also co-stimulatory and engage $\mathrm{T}$ cells, CD54 promotes $\mathrm{DC}-\mathrm{T}$ cell binding and HLA-DR, otherwise known as MHC class II, allows for antigen presentation to $\mathrm{CD} 4+\mathrm{T}$ cells [45-47]. Altered HLA class II cell surface expression, a mechanism by which tumour cells escape from $\mathrm{T}$ cell responses, has been reported in many types of cancer [48]. Normally antigen presentation cells, including DCs, constitutively express HLA class II molecules on the cell membrane, while only minor percentage of tumours and tumour cells express HLA-DR. In this study, we found opposing results for HLA-DR for oesophageal cancer, where the in vitro model inhibited HLA-DR on DCs and the ex vivo model enhanced HLADR on DCs. The reason for the modulation of HLA-DR in oesophageal cancer is unclear. This finding could potentially indicate that the tumour epithelial cells may contribute to the inhibition of HLA-DR, rather than the more complex oesophageal tumour microenvironment which contains both epithelial and non-epithelial cells. Whereas we found that both in vitro and ex vivo models of colonic cancer inhibited HLA-DR. These are contradictory findings and the reasons are unclear, in particular because we have previously shown that HLA-DR expression in tumour

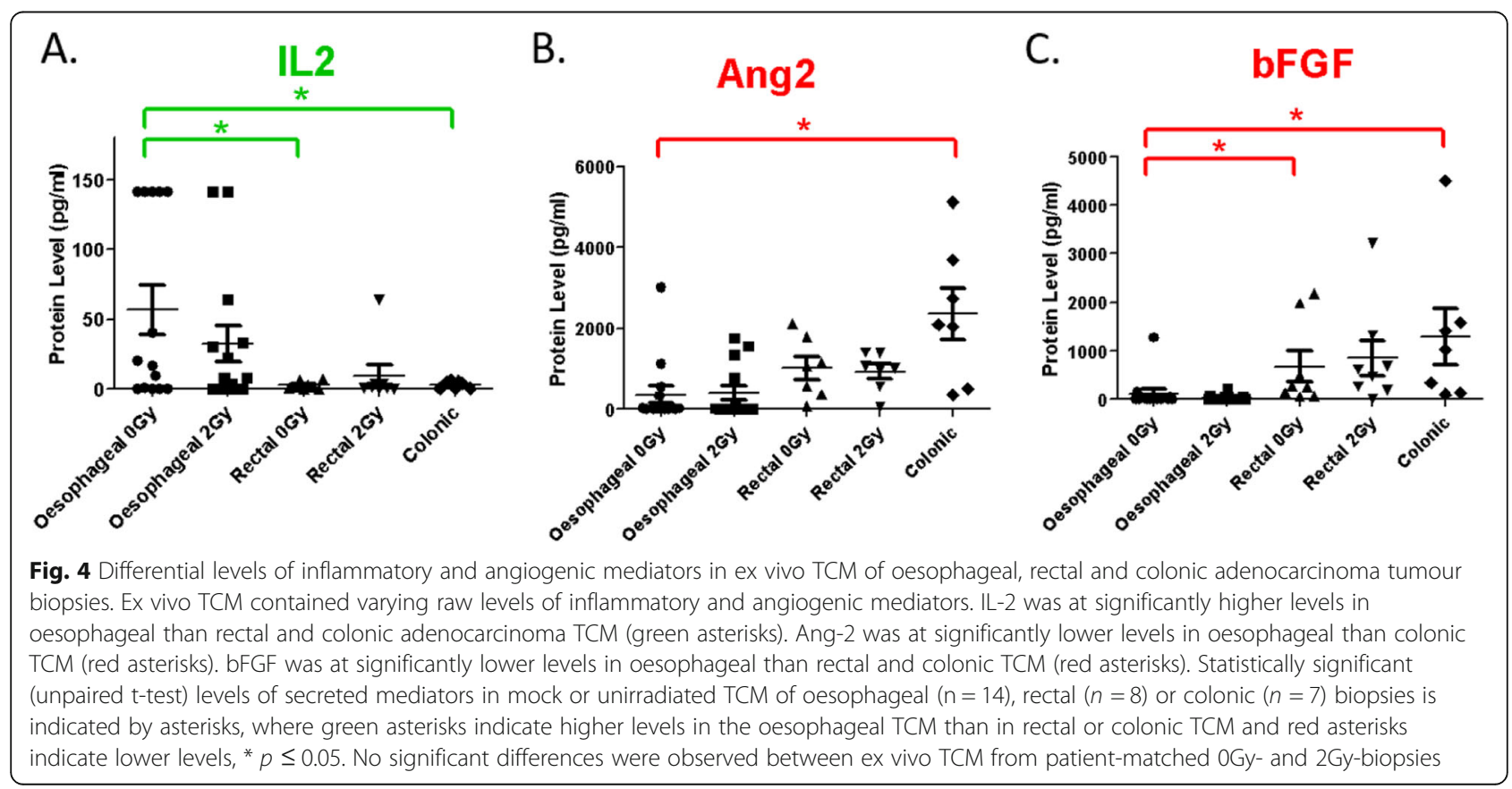


epithelium is an independent prognostic indicator in oesophageal adenocarcinoma patients, and speculate that for patients with enhanced survival, tumour epithelial cells may be compensating for the loss of HLA on antigen presentation cells $[49,50]$.

The SW480 and SW620 cell lines are routinely categorised as colorectal adenocarcinoma [51]. There were minor differences in the effects of SW480 and SW620 cell lines, which are models of primary versus metastatic lesions respectively. While both inhibited three DC markers, SW480 inhibited CD80 and SW620 inhibited CD54. This may suggest that, in addition to the type and localization of the primary GI tumour, but also the nature of the malignant lesion could potentially have a distinct effect on DC function in the TME. Using ex vivo TCM, generated using tumour biopsy explants from oesophageal, rectal and colonic adenocarcinoma, we assessed their differential effects on LPS-induced DC maturation. For DC surface markers in the LPSstimulated setting, oesophageal cancer enhanced five DC markers (CD54, CD80, HLA-DR, CD86 and CD83), rectal cancer enhanced the levels of three DC markers (CD80, CD86 and CD83), whereas colonic cancer inhibited the levels of five DC markers (CD54, HLA-DR, CD86, CD83 and PD-L1) compared to LPS-induced levels in respective background media. Saying that, there was inter-individual variability apparent in the DC maturational capacity induced by ex vivo TCMs from the same GI tract tumour type. In this study, we confirmed the previously reported inhibition of DC markers by colonic adenocarcinoma, and we identified that LPSinduced PD-L1, which has a key role in the resolution of inflammation as the ligand for PD-1, is also inhibited $[18-20,52]$. While the reason is unclear, we speculate that this highlights the poor potential of DCs to respond to maturational stimuli in any capacity in the colonic cancer setting. Immunophenotyping tumour-infiltrating DCs across GI cancers would be important to confirm if this effect occurs in vivo, although quantification of maturation markers, including CD83, can be difficult [53]. In our future studies, additional DC membrane markers will be included such as TLRs and other innate receptors in order to better understand the DC priming phenotypes induced by TCM. While functionally mature DCs are required in order to enable antigen presentation and $\mathrm{T}$ cell clonal expansion, co-culturing TME-conditioned DCs with $\mathrm{T}$ cells would further elucidate the overall functional significance in future studies [20]. Different types of immunosuppressive dendritic cells have been found in cancer patients and animals [54]. Lutz et al., 2002 proposed that tolerance occurs with either partialor semi-maturation of DCs, whereas only full DC maturation is immunogenic and the decisive signal is the release of proinflammatory cytokines from DCs [55].
Interestingly, in vitro conditioned media from both OAC and CRC lines inhibited LPS-induced levels of TNF- $\alpha$ in DC secretions, but had no significant effect on IL-12p70. Similarly, ex vivo TCM from all three GI cancer types significantly inhibited LPS-induced levels of TNF- $\alpha$ in DC secretions. There were differential effects on LPS-induced levels of IL-12p70 in DC supernatants based on adenocarcinoma type with oesophageal having no effect, rectal significantly enhancing, and colonic significantly reducing levels compared to LPS in corresponding background media alone. Thus, we confirmed the known IL-12p70 inhibition and identified that DC TNF- $\alpha$ is also significantly inhibited by the colonic TME $[18,20]$. As TNF- $\alpha$ from DCs is considered immunostimulatory, this then supports an extensive immunosuppressive phenotype induced by the colonic TME, in particular as shown by DC IL-12p70 inhibition, a requirement for optimal anti-tumour immunity $[24,26]$. There is a self-regulatory feedback on DCs of IL-12 and TNF- $\alpha$, which may occur in order to limit the effects of DCs [26]. The notable limitations of this study are the use of a single PBMC donor to derive DCs for each experiment, which was performed to confine interindividual variability to cancer donors, and the limited number of treatment-naïve human tumour biopsies ( $n=8-14$ ). While these need to be addressed to confirm reproducibility of our observations, the finding of TNF- $\alpha$ inhibition occurred across all cancer types and using both in vitro and ex vivo cancer samples. As the finding was common to both in vitro and ex vivo models, this indicates that soluble factors from the tumour epithelial cells may underlie this inhibition, rather than nonepithelial cells of the tumour microenvironment. Our finding of common TNF- $\alpha$ inhibition by GI cancers may have implications for TNF- $\alpha$ blockade, as has been proposed to overcome resistance to anti-PD-1 treatment [56]. Bertrand et al., 2017 proposed that TNF deficiency may favour DC accumulation in tumours, while reducing the expression of PD-1 ligands [56]. In line with this, our findings of reduced TNF- $\alpha$ could potentially indicate accumulation in the GI tract tumour setting of DCs with altered maturational capacities. We have previously shown that the TME of both early- and late-stage colonic cancer is equally suppressive for maturational capacity in DCs [41]. Interestingly, Scarlett et al., 2012 demonstrated that tumour-resident DCs are transformed from immunostimulatory to immunosuppressive during tumour progression in a mouse model of ovarian cancer [57]. Thus if DCs have a dynamic, even immunosuppressive, function in cancer as suggested by our study, then one could speculate from our findings that activation of suboptimally matured DCs in oesophageal adenocarcinoma could potentially result in a poorer outcome than in a setting where DC maturation is more completely suppressed, such as in colonic adenocarcinoma. 
When considering the implications for the findings here, it should be noted that this study is based on a model reflecting only a part of a complex system, specifically it is a model of the humoral components of the TME. These findings support conducting a larger study to determine if a negative correlation exists with outcomes, such as radioresponse and 5-year survival, across GI cancers. A larger study is also required to address some important experimental limitations of this study - in particular that DCs from multiple healthy donors should be employed, additional treatment-naïve tumour samples and non-cancerous matching GI tissues and /or cells as controls.

In this study, 2Gy-irradiation of cell lines and tumour explants was performed to correspond with the physiological effects of radiotherapy treatment at the tissue level as is performed clinically for oesophageal and rectal adenocarcinoma [42]. We found an inhibitory effect of irradiation on DC maturation through conditioned media from both in vitro and ex vivo models. Whether this finding, that TCM from 2Gy-irradiated TME inhibited DC maturation, has relevance to tumour response to radiotherapy warrants further investigation as mentioned above. However, no significant effect was observed with the clinical outcome of tumour regression grade on this small oesophageal and rectal adenocarcinoma patient cohort in this study $(n=14$ and 10 respectively, data not shown). We propose that irradiation of the TME alters release of unknown soluble factors that are discernible to DCs, which fits with a mechanism of the radiation-induced bystander effect via altered levels of inflammatory cytokines produced by the TME [5862]. However, the irradiated TCMs had no effect on DC secretion levels of TNF- $\alpha$ or IL-12p70, therefore the functional significance of the effect on DC markers is difficult to decipher in terms of the ability of DCs to potentiate any immunomodulatory message to other bystander cells. Inflammation and angiogenesis are closely related and may underlie immune inhibition and radioresponse [20]. Unfortunately, profiling the ex vivo TCM for inflammatory and angiogenic factors did not identify differences in levels of any of the mediators between 0Gy- and 2Gy-irradiated patient-matched biopsies. Radiotherapy has been described as both immunostimulatory and immunosuppressive with radiation dose proposed to be a key influencer in this, where low dose radiation, such as $2 \mathrm{~Gy}$, may be immunosuppressive [32, 37, 63]. This data could suggest that radiotherapy of the GI TME may further reduce DC maturation, which we speculate could be beneficial in improving outcome in a setting where DCs may only have the capacity to sub-optimally mature, such as oesophageal adenocarcinoma.

As tumour biopsies contain tumour epithelial cells in addition to other cell types, ex vivo TCM contains many different tumour associated soluble factors, and therefore closely mimics the inflammatory milieu of the tumour in situ. Several cytokines and chemokines have been described to be present at high levels in the colonic TME compared to normal tissues, such as CXCL1 and CXCL5 (which function to attract and activate neutrophils) and CCL2 (a chemoattractant for monocytes, memory T cells and DCs) [20]. We do not yet know the mechanistic pathways or secreted factors that induce the DC phenotypes we observed. Although NF-кB would be a candidate pathway given its reported roles in both LPS-induced DC maturation that ultimately results in the activation of NF- $\mathrm{NB}$ and the production of proinflammatory cytokines and in radiation-triggered TNF- $\alpha-N F-\kappa B$ cross-signalling [62, $64,65]$. In this study, levels of specific inflammatory and angiogenic mediators in ex vivo TCM of oesophageal, rectal and colonic adenocarcinoma were correlated with DC maturation marker - CD54. Thus it is possible that low levels of the cytokine IL-2 and high levels of angiogenic mediators - Ang-2 and bFGF, in TCM of tumour biopsies may confer a more DC inhibitory environment and this fits with some expected roles for these mediators. As CD54 (also known as intercellular adhesion molecule 1, ICAM-1) promotes DC-T cell binding, this indicates possible negative effects on the capacity of DCs to activate T cells in order to induce an adaptive immune response in GI tumours with lower levels of IL-2 and higher levels of Ang-2 and bFGF. IL-2 has key functions in the immune system, tolerance and immunity, primarily via direct effects on $\mathrm{T}$ cells, both effector and regulatory type. Interestingly, a role has been proposed for DC maturation as a mediator of systemic IL-2 effects [66]. DCs can differentiate into endothelial-like cells when cultured in the presence of angiogenic growth factors - bFGF, VEGF and IGF-1, and these altered DCs have a reduced functional potency [67]. We have previously shown that levels of tumour vasculature maturity or DC inhibition negatively correlate with survival of colonic adenocarcinoma patients on anti-angiogenic treatment $[40,68]$. We have previously identified that multiple mediators influence DC inhibition in colonic TCM - increasing levels of CXCL1, CXCL5, CCL2 and VEGF in the TCM correlated with inhibition of IL-12p70 secretion from DCs, however these isolated factors were not sufficient to induce all aspects of the extensive DC inhibition as observed for colonic TCM [20]. The data from this study further supports the concept that it may be the cumulative effect of many mediators in the TME that may influence DCs. In particular, the levels of TGF-beta may be relevant due to its pivotal role in inducing immunological tolerance in DCs in colonic tissue [69, 70]. While the effects of distinct ex vivo TCMs on DCs are described relative to their corresponding background media, profiling the levels of mediators may not be representative of the in vivo setting due to differences in culturing conditions. In future studies, healthy tissue controls 


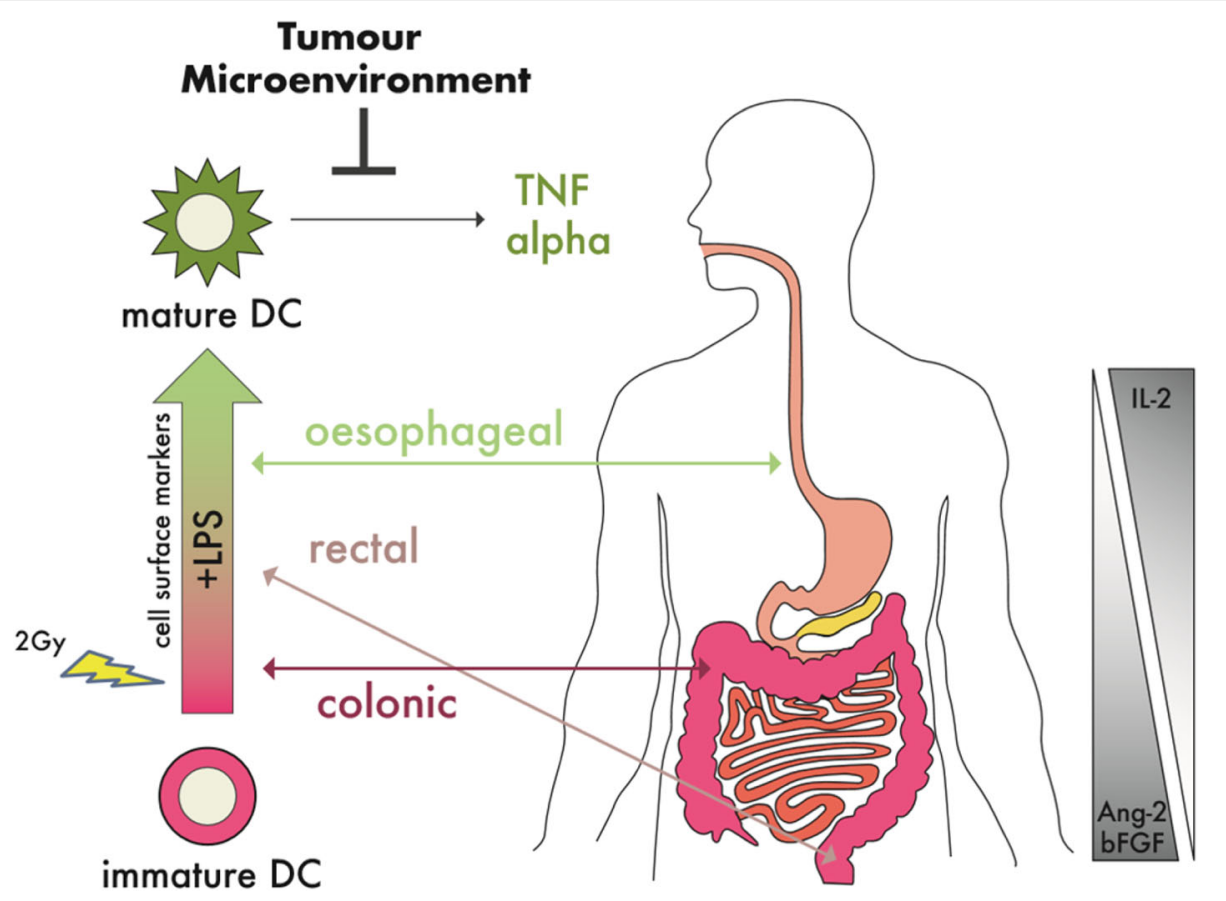

Fig. 5 While there was varying effects on DC maturational capacity induced by distinct gastrointestinal adenocarcinoma types, oesophageal, rectal and colonic TME inhibited DC secreted TNF-a. This model summarises that treatment of DCs with GI TCM revealed differential effects on DC maturational capacity with oesophageal cancer enhancing most markers, rectal cancer enhancing three markers and with colonic cancer inhibiting most markers. In addition, 2Gy-irradiation of the TME inhibited LPS-induced levels of DC markers. Differential levels of angiogenic and inflammatory mediators in ex vivo TMEs correlated with effects on DC surface markers, with IL-2 positively correlating and with Ang2 and bFGF negatively correlating with the DC maturation marker CD54. Regardless of the effect on DC surface markers, the TME of all GI tract cancer types significantly inhibited DC secreted TNF-a levels

and cells will be examined in conjunction with cancerous samples. GI cancers are well known to be molecularly heterogeneous with, for example, a substantial proportion of tumours (15\% of CRC) displaying microsatellite instability phenotype and these tumours are commonly highly immunogenic, densely infiltrated with activated $\mathrm{T}$ cells and respond well to immune checkpoint blockade therapy [71]. Therefore it would be interesting to examine if this subset of CRC tumours are not able to inhibit DC maturation to the same extent as observed in this study for which we do not know the MSI phenotype of the tumour biopsies.

\section{Conclusions}

In conclusion, this study demonstrates for the first time that there are differences in the levels of DC maturational capacity across distinct human GI adenocarcinomas (Fig. 5). The consistent and significant inhibition of DC TNF- $\alpha$ across oesophageal, rectal and colonic adenocarcinoma may be the key way in which DC maturation is dysregulated by GI cancers. In addition, radiotherapy-mimicked TCM further inhibited DC maturational capacity at the maturation marker level. Differential levels of secreted mediators of inflammation (IL-2) and angiogenesis (Ang2 and bFGF) in the TME may underlie variation in DC responsiveness. This in turn may reduce the capacity of localised DCs to induce an anti-tumour immune response and may have implications for response to radiotherapy. As the oesophageal TME appears to be less inhibitory compared to colonic adenocarcinoma, this may have implications for informing immunotherapy, such as DC vaccines, and PD-1/PDL1 or TNF- $\alpha$ blockade [56, 72, 73]. Indeed, LPS-induced levels of PD-L1 on TME-conditioned DCs was only inhibited by colonic adenocarcinoma, but not oesophageal or rectal adenocarcinoma. This study warrants further functional and in vivo investigations as it may have implications for how localised DCs are inhibited from inducing anti-tumour immunity in GI cancer and may also influence response to radiotherapy.

\section{Supplementary information}

Supplementary information accompanies this paper at https://doi.org/10. 1186/s12885-020-07012-y.

Additional file 1 Supplementary Table 1. Patient demographics. Supplementary Fig. 1. Experimental outline and flow cytometry gating strategy and staining controls. Supplementary Fig. 2. ex vivo TCM from distinct gastrointestinal adenocarcinoma types induced differential effects on LPS-induced DC maturation. Supplementary Fig. 3. ex vivo TCM from distinct gastrointestinal adenocarcinoma types induced differential 
effects on unstimulated DC marker levels. Supplementary Fig. 4. in vitro TCM of 2Gy-irradiated cell lines from gastrointestinal cancers induced significant inhibition of DC markers compared to mock irradiation. Supplementary Fig. 5. ex vivo TCM of 2Gy-irradiated TME from gastrointestinal cancers inhibited DC markers compared to mock irradiation

\section{Abbreviations}

CD: Cluster of Differentiation; CM199: Complete Medium 199; CRC: Colorectal Cancer; cRPMI: Complete Roswell Park Memorial Institute 1640 Medium; CRT: Chemoradiotherapy; DC: Dendritic Cell; ELISA: Enzyme-Linked Immunosorbent Assay; FMO: Fluorescence Minus One; Gl: Gastrointestinal; Gy: Gray; HLA-DR: Major Histocompatibility Complex, Class II, DR; IL: Interleukin; LPS: Lipopolysaccharides; OAC: Oesophageal Adenocarcinoma; PBMCs: Peripheral Blood Mononuclear Cells; PD-L1: Programmed Cell Death 1-Ligand 1; TCM: Tumour Conditioned Media; TME: Tumour Microenvironment; TNF-a: Tumor Necrosis Factor-Alpha

\section{Acknowledgments}

The authors would like to acknowledge patients for consenting to provide samples and Irish Blood Transfusion Service, St James's Hospital for providing buffy coats. Miriam Tosetto, UCD for providing CRC cell lines. Paul Quinlan, St James' Hospital for graphic design

\section{Authors' contributions}

MEM, JOS conceived and designed the experiments with input from JL, JVR, $E R, M D$. MEM and RB performed the experiments with assistance from CN, NM, SK. DO, JL, PM, BM provided human samples; NLL, CN provided cell line samples; CB, MC provided colonic tissue conditioned media. MEM, JOS wrote the paper. The authors approved the manuscript.

\section{Funding}

This study was funded by a research fellowship (CRF13MOR) provided by the Irish Cancer Society to Maria E. Morrissey. The funding body did not have a role in the design of the study and collection, analysis and interpretation of data or writing of the manuscript.

\section{Availability of data and materials}

The datasets used and/or analysed during the current study are available from the corresponding author on reasonable request.

\section{Ethics approval and consent to participate}

All procedures performed in studies involving human participants were in accordance with the ethical standards of the institutional research committee (Adelaide and Meath Hospital Research Ethics Committee) and with the 1964 Helsinki declaration and its later amendments or comparable ethical standards. Written informed consent was obtained from all individual participants included in the study.

\section{Consent for publication}

Not applicable.

\section{Competing interests}

The authors declare that they have no conflict of interest.

\section{Author details}

'Department of Surgery, Trinity Translational Medicine Institute, Trinity College Dublin, St James's Hospital, Dublin 8, Ireland. ${ }^{2}$ UCD School of Biomolecular and Biomedical Sciences, UCD Conway Institute, University College Dublin, Belfield, Dublin 4, Ireland. ${ }^{3}$ Department of Clinical Medicine, Trinity Translational Medicine Institute, Trinity College Dublin, St. James's Hospital, Dublin 8, Ireland. ${ }^{4}$ GEMS, St James's Hospital, Dublin, Ireland. ${ }^{5}$ Oesophageal Unit, St James's Hospital, Trinity College Dublin, Dublin, Ireland. ${ }^{6}$ Centre for Colorectal Disease, Education and Research Centre, St Vincent's University Hospital, Elm Park, Dublin 4, Ireland. 'Department of Biological Sciences, Health Research Institute, University of Limerick, Castletroy, Co., Limerick, Ireland.
Received: 30 January 2020 Accepted: 27 May 2020

Published online: 17 June 2020

\section{References}

1. National Cancer Registry Ireland; 2018. https:/www.ncri.ie/.

2. Enzinger PC, Mayer RJ. Esophageal cancer. N Engl J Med. 2003;349(23):224152.

3. Glimelius B. Neo-adjuvant radiotherapy in rectal cancer. World J Gastroenterol. 2013;19(46):8489-501.

4. Glimelius B, et al. Rectal cancer: ESMO clinical practice guidelines for diagnosis, treatment and follow-up. Ann Oncol. 2013;24 Suppl 6:vi81-8.

5. Lloyd S, Chang BW. Current strategies in chemoradiation for esophageal cancer. J Gastrointest Oncol. 2014;5(3):156-65.

6. Onaitis MW, et al. Neoadjuvant chemoradiation for rectal cancer: analysis of clinical outcomes from a 13-year institutional experience. Ann Surg. 2001; 233(6):778-85.

7. Carrato A. Adjuvant treatment of colorectal cancer. Gastrointest Cancer Res. 2008:2(4 Suppl):S42-6.

8. Walsh TN, et al. A comparison of multimodal therapy and surgery for esophageal adenocarcinoma. N Engl J Med. 1996;335(7):462-7.

9. Geh JI, Crellin AM, Glynne-Jones R. Preoperative (neoadjuvant) chemoradiotherapy in oesophageal cancer. Br J Surg. 2001:88(3):338-56.

10. Luu TD, et al. Neoadjuvant chemoradiation versus chemotherapy for patients undergoing esophagectomy for esophageal cancer. Ann Thorac Surg. 2008;85(4):1217-23 discussion 1223-4.

11. Das $\mathrm{P}$, et al. Clinical and pathologic predictors of locoregional recurrence, distant metastasis, and overall survival in patients treated with chemoradiation and mesorectal excision for rectal cancer. Am J Clin Oncol. 2006;29(3):219-24

12. Das $\mathrm{P}$, et al. Predictors of tumor response and downstaging in patients who receive preoperative chemoradiation for rectal cancer. Cancer. 2007;109(9): 1750-5.

13. Park IJ, et al. Neoadjuvant treatment response as an early response indicator for patients with rectal cancer. J Clin Oncol. 2012;30(15):1770-6.

14. Steel GG. The ESTRO Breur lecture. Cellular sensitivity to low dose-rate irradiation focuses the problem of tumour radioresistance. Radiother Oncol. 1991;20(2):71-83.

15. McMillan TJ, et al. The relationship of DNA double-strand break induction to radiosensitivity in human tumour cell lines. Int J Radiat Biol. 1990:58(3):42738

16. Zaffaroni $\mathrm{N}$, et al. DNA double-strand break repair and radiation response in human tumour primary cultures. Int J Radiat Biol. 1994;66(3):279-85.

17. Mellman I, Steinman RM. Dendritic cells: specialized and regulated antigen processing machines. Cell. 2001;106(3):255-8.

18. Michielsen AJ, O'Sullivan JN, Ryan EJ. Tumor conditioned media from colorectal cancer patients inhibits dendritic cell maturation. Oncoimmunology. 2012;1 (5):751-3.

19. Unger WW, et al. Induction of Treg by monocyte-derived DC modulated by vitamin D3 or dexamethasone: differential role for PD-L1. Eur J Immunol. 2009:39(11):3147-59.

20. Michielsen AJ, et al. Tumour tissue microenvironment can inhibit dendritic cell maturation in colorectal cancer. PLoS One. 2011:6(11):e27944.

21. Pinzon-Charry A, Maxwell T, Lopez JA. Dendritic cell dysfunction in cancer: a mechanism for immunosuppression. Immunol Cell Biol. 2005;83(5):451-61.

22. Corinti $\mathrm{S}$, et al. Regulatory activity of autocrine IL-10 on dendritic cell functions. J Immunol. 2001:166(7):4312-8.

23. Minkis $\mathrm{K}$, et al. Type 2 bias of $\mathrm{T}$ cells expanded from the blood of melanoma patients switched to type 1 by IL12 p70 mRNA-transfected dendritic cells. Cancer Res. 2008;68(22):9441-50.

24. Xu S, et al. High-avidity antitumor T-cell generation by toll receptor 8primed, myeloid- derived dendritic cells is mediated by IL 12 production. Surgery. 2006;140(2):170-8.

25. Tian $\mathrm{L}$, et al. Growth suppression of human laryngeal squamous cell carcinoma by adenoviral-mediated interleukin-12. J Int Med Res. 2010;38(3): 994-1004.

26. Ebrahimi M, et al. Immediate exposure to TNF-a activate dendritic cells derived from non-purified cord blood mononuclear cells. Iran J Immunol. 2009;6(3):107-18.

27. Xu S, et al. Rapid high efficiency sensitization of CD8+ T cells to tumor antigens by dendritic cells leads to enhanced functional avidity and direct 
tumor recognition through an IL12-dependent mechanism. J Immunol. 2003;171(5):2251-61.

28. Ryan AM, et al. Adenocarcinoma of the oesophagus and gastric cardia: male preponderance in association with obesity. Eur J Cancer. 2006:42(8):1151-8.

29. Renehan $A G$, et al. Body-mass index and incidence of cancer: a systematic review and meta-analysis of prospective observational studies. Lancet. 2008; 371(9612):569-78

30. Triantafillidis JK, Nasioulas G, Kosmidis PA. Colorectal cancer and inflammatory bowel disease: epidemiology, risk factors, mechanisms of carcinogenesis and prevention strategies. Anticancer Res. 2009;29(7):2727-37.

31. Multhoff $G$, Radons J. Radiation, inflammation, and immune responses in cancer. Front Oncol. 2012;2:58.

32. Ahmed MM, et al. Immunobiology of radiotherapy: new paradigms. Radiat Res. 2014;182(2):123-5.

33. Shigematsu $A$, et al. Effects of low-dose irradiation on enhancement of immunity by dendritic cells. J Radiat Res. 2007:48(1):51-5.

34. Merrick A, et al. Immunosuppressive effects of radiation on human dendritic cells: reduced IL12 production on activation and impairment of naive T-cell priming. Br J Cancer. 2005;92(8):1450-8.

35. McBride WH, et al. A sense of danger from radiation. Radiat Res. 2004;162(1):1-19.

36. Finkelstein $\mathrm{SE}$, et al. Combination of external beam radiotherapy (EBRT) with intratumoral injection of dendritic cells as neo-adjuvant treatment of highrisk soft tissue sarcoma patients. Int J Radiat Oncol Biol Phys. 2012;82(2): 924-32.

37. Roses RE, Datta J, Czerniecki BJ. Radiation as immunomodulator: implications for dendritic cell-based immunotherapy. Radiat Res. 2014; 182(2):211-8.

38. Garg AD, et al. Integrating next-generation dendritic cell vaccines into the current cancer immunotherapy landscape. Trends Immunol. 2017;38(8):577-93.

39. Michielsen AJ, Ryan EJ, O'Sullivan JN. Dendritic cell inhibition correlates with survival of colorectal cancer patients on bevacizumab treatment. Oncoimmunology. 2012;1(8):1445-7.

40. Michielsen AJ, et al. Inhibition of dendritic cell maturation by the tumor microenvironment correlates with the survival of colorectal cancer patients following bevacizumab treatment. Mol Cancer Ther. 2012;11(8):1829-37.

41. O'Toole A, et al. Tumour microenvironment of both early- and late-stage colorectal cancer is equally immunosuppressive. Br J Cancer. 2014;111(5): 927-32.

42. Lynam-Lennon $\mathrm{N}$, et al. Alterations in DNA repair efficiency are involved in the radioresistance of esophageal adenocarcinoma. Radiat Res. 2010;174(6): 703-11.

43. Dunne MR, et al. (E)-4-hydroxy-3-methyl-but-2 enyl pyrophosphatestimulated Vgamma9Vdelta2 T cells possess T helper type 1-promoting adjuvant activity for human monocyte-derived dendritic cells. Cancer Immunol Immunother. 2010;59(7):1109-20.

44. Dong $\mathrm{G}$, et al. FOXO1 regulates dendritic cell activity through ICAM-1 and CCR7. J Immunol. 2015;194(8):3745-55.

45. Prechtel AT, Steinkasserer A. CD83: an update on functions and prospects of the maturation marker of dendritic cells. Arch Dermatol Res. 2007;299(2):59-69.

46. Flores-Romo L. In vivo maturation and migration of dendritic cells. Immunology. 2001;102(3):255-62

47. Brooks CF, Moore M. Differential MHC class II expression on human peripheral blood monocytes and dendritic cells. Immunology. 1988;63(2): 303-11.

48. Mendez R, et al. HLA and melanoma: multiple alterations in HLA class I and II expression in human melanoma cell lines from ESTDAB cell bank. Cancer Immunol Immunother. 2009;58(9):1507-15.

49. Dunne MR, et al. HLA-DR expression in tumor epithelium is an independent prognostic indicator in esophageal adenocarcinoma patients. Cancer Immunol Immunother. 2017;66(7):841-50.

50. Dunne MR, et al. Characterising the prognostic potential of HLA-DR during colorectal cancer development. Cancer Immunol Immunother. 2020 Apr 18. https://doi.org/10.1007/s00262-020-02571-2.

51. Hewitt RE, et al. Validation of a model of colon cancer progression. J Pathol. 2000;192(4):446-54.

52. Brown JA, et al. Blockade of programmed death-1 ligands on dendritic cells enhances T cell activation and cytokine production. J Immunol. 2003;170(3): 1257-66.

53. Dzionek A, et al. BDCA-2, BDCA-3, and BDCA-4: three markers for distinct subsets of dendritic cells in human peripheral blood. J Immunol. 2000; 165(11):6037-46.
54. Shurin GV, Ma Y, Shurin MR. Immunosuppressive mechanisms of regulatory dendritic cells in cancer. Cancer Microenviron. 2013;6(2):159-67.

55. Lutz MB, Schuler G. Immature, semi-mature and fully mature dendritic cells: which signals induce tolerance or immunity? Trends Immunol. 2002;23(9): 445-9.

56. Bertrand F, et al. TNFa blockade overcomes resistance to anti-PD-1 in experimental melanoma. Nat Commun. 2017;8(1):2256.

57. Scarlett UK, et al. Ovarian cancer progression is controlled by phenotypic changes in dendritic cells. J Exp Med. 2012;209(3):495-506.

58. Marin A, et al. Bystander effects and radiotherapy. Rep Pract Oncol Radiother. 2015:20(1):12-21.

59. Najafi $M$, et al. The mechanisms of radiation-induced bystander effect. J Biomed Phys Eng. 2014;4(4):163-72

60. Liu SZ, Jin SZ, Liu XD. Radiation-induced bystander effect in immune response. Biomed Environ Sci. 2004:17(1):40-6.

61. Calveley $\mathrm{VL}$, et al. Partial volume rat lung irradiation: temporal fluctuations of in-field and out-of-field DNA damage and inflammatory cytokines following irradiation. Int J Radiat Biol. 2005;81(12):887-99.

62. Veeraraghavan J, et al. Radiation-triggered tumor necrosis factor (TNF) alpha-NFkappaB cross-signaling favors survival advantage in human neuroblastoma cells. J Biol Chem. 2011;286(24):21588-600.

63. Jahns J, et al. Influence of low dose irradiation on differentiation, maturation and T-cell activation of human dendritic cells. Mutat Res. 2011;709-710:32-9.

64. Hirschfeld M, Ma Y, Weis JH, Vogel SN, Weis JJ. Cutting edge: repurification of lipopolysaccharide eliminates signaling through both human and murine toll-like receptor 2. J Immunol. 2000 Jul 15;165(2):618-22.

65. Ade $\mathrm{N}$, et al. NF-kappaB plays a major role in the maturation of human dendritic cells induced by NiSO (4) but not by DNCB. Toxicol Sci. 2007;99(2): 488-501.

66. Nagorsen D, Marincola FM, Panelli MC. Cytokine and chemokine expression profiles of maturing dendritic cells using multiprotein platform arrays. Cytokine. 2004;25(1):31-5.

67. Fernandez Pujol B, et al. Dendritic cells derived from peripheral monocytes express endothelial markers and in the presence of angiogenic growth factors differentiate into endothelial-like cells. Eur J Cell Biol. 2001;80(1):99110.

68. Noonan SA, et al. Tumour vasculature immaturity, oxidative damage and systemic inflammation stratify survival of colorectal cancer patients on bevacizumab treatment. Oncotarget. 2018:9(12):10536-48.

69. Esebanmen GE, Langridge WHR. The role of TGF-beta signaling in dendritic cell tolerance. Immunol Res. 2017;65(5):987-94.

70. Ihara $\mathrm{S}$, et al. TGF- $\beta$ signaling in dendritic cells governs colonic homeostasis by controlling epithelial differentiation and the luminal microbiota. J Immunol. 2016;196(11):4603-13.

71. Kloor M, et al. Clinical significance of microsatellite instability in colorectal cancer. Langenbecks Arch Surg. 2014;399(1):23-31.

72. Kalinski $P$, et al. Dendritic cell-based therapeutic cancer vaccines: what we have and what we need. Future Oncol. 2009:5(3):379-90.

73. Brossart $P$, et al. Induction of cytotoxic T-lymphocyte responses in vivo after vaccinations with peptide-pulsed dendritic cells. Blood. 2000;96(9):3102-8.

\section{Publisher's Note}

Springer Nature remains neutral with regard to jurisdictional claims in published maps and institutional affiliations.

Ready to submit your research? Choose BMC and benefit from:

- fast, convenient online submission

- thorough peer review by experienced researchers in your field

- rapid publication on acceptance

- support for research data, including large and complex data types

- gold Open Access which fosters wider collaboration and increased citations

- maximum visibility for your research: over $100 \mathrm{M}$ website views per year

At $\mathrm{BMC}$, research is always in progress.

Learn more biomedcentral.com/submissions 